\title{
Strong Electronic Coupling of Graphene Nanoribbons onto Basal Plane of Glassy Carbon Electrode
}

\author{
Marija R. Zoric, ${ }^{1,2}$ Erik J. Askins, ${ }^{1,2}$ Xiaoxiao Qiao, ${ }^{1,2}$ Ksenija D. Glusac ${ }^{1,2 *}$ \\ ${ }^{1}$ Department of Chemistry, University of Illinois at Chicago, Chicago, Illinois 60607 \\ ${ }^{2}$ Chemical Sciences and Engineering Division, Argonne National Laboratory, Lemont, Illinois 60439
}

\begin{abstract}
The grafting of molecular motifs to the conductive carbon represents a promising approach towards new hybrid materials for electrocatalytic applications. Here, we investigate the electrochemical behavior of graphene nanoribbons deposited onto glassy carbon electrode using $\pi-\pi$ stacking interactions. Using the bipyrimidine moiety on the nanoribbon edges as a reporter of the proton-coupled electron transfer chemistry, we illustrate that the simple electrochemical treatment of as-deposited nanoribbon generates a hybrid material that is in strong electronic communication with the conductive support. This work shows novel strategy for modifying basal plane of carbon materials and provide potential platform for incorporation of catalytic metal sites via coordination through N-functionalities of GNR.
\end{abstract}

Graphitic nanocarbon materials have recently been recognized as promising electrocatalysts for a range of useful transformations, such as the reduction of dioxygen to water, dinitrogen to ammonia, and carbon dioxide to methanol. ${ }^{1-3}$ The success of these electrode materials is attributed to the high electronic conductivity of graphitic carbon, the high surface area of nanocarbon electrodes made using template-directed pyrolysis, and the presence of reactive catalytic functional groups obtained by heteroatom doping of otherwise chemically inert carbon framework. Further progress in the field requires the synthesis of nanocarbon electrodes with complex multinuclear catalytic motifs that go beyond "single-atom catalysts". Unfortunately, introduction of such well-defined catalytic functionalities is limited by the extreme temperatures required to graphitize carbon precursors, at which most functional groups undergo decomposition.

A more favorable way to introduce catalytic moieties to graphitic electrodes involves post-pyrolysis modifications of carbon edge and basal sites with well-defined molecular motifs. For this purpose, several creative chemical methods have been developed to immobilize molecular catalysts to the carbonbased surfaces using covalent bonds, ${ }^{4-11} \pi-\pi$ stacking ${ }^{12-19}$ and electrostatic interactions. ${ }^{20,21}$ Carbon edge sites are often modified covalently using 'click' chemistry ${ }^{4,7}$ or aryl radical intermediates formed from diazonium salts. ${ }^{5,6}$ Carbon basal sites are modified using $\pi-\pi$ stacking interactions between polyaromatic groups, most commonly pyrene, and the carbon electrode. Here, the catalytic units are connected to pyrene through an alkyl chain. ${ }^{15-17,19}$ These modification techniques ensure the immobilization of homogeneous catalysts, thus eliminating the need for their diffusion to the electrode surface. However, the electrochemical behavior of such immobilized catalysts usually does not differ from that observed in homogeneous analogs because the electronic coupling between the catalytic moieties and the conductive carbon electrode remains weak.
More recently, chemical methods have been explored to graft molecular catalysts using strong electronic coupling to create new hybrid structures with altered reactivity and catalytic behavior. Specifically, Compton and coworkers showed that the carbon edge planes can be functionalized using ortho-quinone groups generated by the anodic treatment of the electrode surface. ${ }^{22}$ The Surendranath group used this coupling method to attach molecular catalysts to the carbon edge sites and found that the strong electronic coupling exists between the catalytic unit and the carbon band structures. ${ }^{23-25}$ Such strong coupling resulted in interesting changes of the electrochemical behavior of the grafted hybrid relative to the homogeneous analog. For example, grafted hybrids were shown not to undergo simple outer-sphere electron transfer processes, which in turn had important implications in catalysis by enabling the proton-coupled chemistry to take place: a hydrogen-evolving Rh-based catalyst, when grafted to the carbon electrode, operates over a full $\mathrm{pH}$ region (0-14), whereas the homogeneous analog shows catalytic activity only in the acidic region. ${ }^{23-25}$

Similar mechanistic studies involving basal plane modifications and the strong electronic coupling regime are scarcer. Several recent reports involving immobilized metal-coordinated $\mathrm{N}_{4}$-macrocycles indicate that the $\pi$-stacking interactions may generate strongly coupled hybrids. ${ }^{26-34}$ For example, the coupling of molecular cobalt phthalocyanine, a carbon dioxide reduction catalyst, to a carbon nanotube support has been shown to affect product selectivity: two-electron reduction to $\mathrm{CO}$ is favored when the homogenous catalyst is used, while a six-electron reduction to methanol takes place with the heterogeneous catalyst/carbon hybrid. ${ }^{26}$ Similar studies with immobilized metal-coordinated $\mathrm{N}_{4}$-macrocycles have shown better efficiency toward $\mathrm{CO}_{2}$ reduction to $\mathrm{CO},{ }^{27-32}$ as well as improved nitrogen reduction reaction efficiency. ${ }^{33}$ However, most of those basal plane modification studies have been focused on catalytic performance rather than mechanistic investigation of their fundamental electrochemistry. 

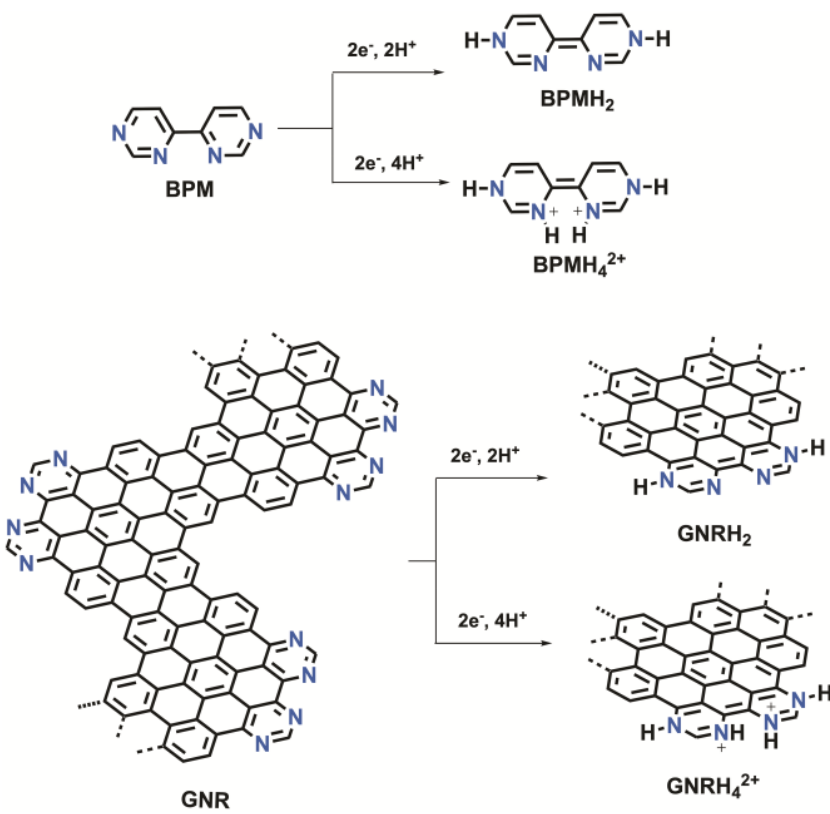

Scheme 1. Structures of model compounds BPM an GNR and the products of their cathodic reduction via two-electron, twoproton $\left(\mathrm{BPMH}_{2}\right.$ and $\left.\mathrm{GNRH}_{2}\right)$ and two-electron four-proton $\left(\mathrm{BPMH}_{4}{ }^{2+}\right.$ and $\left.\mathrm{GNRH}_{4}{ }^{2+}\right)$ transfer chemistry.

Here, we investigate the degree of electronic coupling in molecule/carbon electrode hybrids functionalized using $\pi$-stacking interactions. Specifically, we explore how the electrochemical behavior of 4,4'-bipyrimidine (BPM) changes when immobilized to the glassy carbon surface using graphene nanoribbon linkers (GNR, Scheme 1). GNRs exhibit large planar aromatic structures capable of $\pi-\pi$ stacking with the basal sites of the carbon electrode, while the redox activity of bipyrimidine functionalities reports on the degree of electronic coupling between the nitrogen sites and the electrode surface. Our detailed studies of proton-coupled electron transfer (PCET) chemistry of nitrogen moieties reveal that the electrochemically treated GNRs form monolayers that are strongly coupled with the carbon electrode. This work shows a novel strategy for modifying the basal plane of carbon materials and provides a potential platform for incorporation of catalytic metal sites via coordination through $\mathrm{N}$-functionalities of GNR.

GNR was synthesized according to the method published by Sinitskii and coworkers (SI, Scheme S1). ${ }^{35}$ The procedure involves the synthesis of a soluble aryl-substituted benzene monomer 8 via Diels-Alder cycloaddition/decarbonylation sequence developed by Müllen and co-workers. ${ }^{36}$ Dibrominated monomer 8 was then subjected to Yamamoto coupling to produce soluble polymer 9 . The MALDI mass spectrum of polymer 9 reveals a series of oligomer peaks, separated by 522 au (corresponding to the mass of one monomer unit), with sizes up to 16 monomer units corresponding to $20 \mathrm{~nm}$ length (Figure S1, SI). The sample of polymer 9 may contain longer chains that are not detectable by mass spectrometry, and this hypothesis is consistent with previous reports of micrometer-long nanoribbons obtained using similar Yamamoto coupling procedures. ${ }^{35,37}$ The oxidative cyclodehydrogenation of polymer 9 yielded insoluble GNR, which was characterized using solid state NMR, Raman spectroscopy, transmission electron microscopy, and X-ray photoelectron spectroscopy (XPS) (Figures S2S5, SI).

Electrochemical behavior of BPM was studied using cyclic voltammetry (CV) in aqueous medium (black traces in Figure 1 and Figure S6). Chemically reversible or quasi-reversible reduction features were observed in the cathodic region $(-0.5$ to 0 $\mathrm{V}$ vs. NHE), and the half-wave potential was found to shift to more negative values with increasing $\mathrm{pH}$, indicative of PCET. The equilibrium potentials were used to construct the Pourbaix diagram in Figure 2A, which shows two distinct $\mathrm{pH}$ regions $(0$ 4 and 4-14) in which the potential varies linearly with $\mathrm{pH}$. The experimental data were fit to the Nernst equation for PCET, ${ }^{38}$ which lead to the assignment of the two $\mathrm{pH}$ regions as follows: the $\mathrm{pH}=4-14$ process was assigned to a two-electron two-proton transfer reduction to form $\mathrm{BPMH}_{2}$, while the acidic $\mathrm{pH}=0-4$ region was assigned to the two-electron four-proton coupled reduction to form $\mathrm{BPMH}_{4}{ }^{2+}$ (Scheme 1). This conclusion was further supported by an excellent match between the standard reduction potential $E_{P C E T}^{0}$ obtained from the Pourbaix diagram and the same parameter calculated using standard reduction potentials for single electron transfer processes and the relevant pKa values (more information is available in Tables S1 and S2 of Section S4B, Supporting Information, SI).

The electrochemical behavior of as-deposited GNR is significantly different from BPM (red traces in Figure 1 and Figures $\mathrm{S} 7$ and S8). For example, the $\mathrm{CV}$ of GNR collected at $\mathrm{pH}=1$ exhibits an intense cathodic peak at $\mathrm{E}_{\mathrm{c}}=-0.752 \mathrm{~V}$ and a weaker anodic peak at $E_{a}=1.074 \mathrm{~V}$. This large separation between the cathodic and anodic peaks indicates a large kinetic barrier for the observed electrochemical transformation. Interestingly the approximate half-wave potential for GNR is similar $\left(E_{1 / 2}=202\right.$ $\mathrm{mV})$ to that observed in BPM $\left(\mathrm{E}_{1 / 2}=164 \mathrm{mV}\right)$. Furthermore, the halfwave potential shifts to more negative potentials with increasing $\mathrm{pH}$, suggesting that the observed transformation is the proton-coupled reduction of bipyrimidine moieties in GNR. The slow rate for the observed PCET is likely associated with the low electron and proton conductivity in GNR-aggregates in the as-deposited sample. Similar sluggish kinetics were observed in studies of phthalocyanine aggregates ${ }^{29,39}$ and these results illustrate the need for the development of deposition methods that ensure monomolecular coverage of molecular units and good electronic communication with the carbon support.

Interestingly, the cathodic $\left(\mathrm{I}_{\mathrm{c}}\right)$ and anodic $\left(\mathrm{I}_{\mathrm{a}}\right)$ peak currents in as-deposited GNR are not the same. For example, $\mathrm{I}_{\mathrm{c}} / \mathrm{I}_{\mathrm{a}}$ in the $\mathrm{CV}$ of $\mathrm{GNR}$ at $\mathrm{pH}=1$ is 7, indicating that some of the GNR material is detached from the electrode surface upon reduction. This prompted us to investigate the electrochemical behavior of GNR upon subsequent scans (blue traces in Figure 1 and Figures S7-S9). The CVs of GNR after the first CV scan are significantly different from those for as-deposited GNR. Namely, the second-scan CVs show the appearance of new reversible and $\mathrm{pH}$-dependent features in the 0 to $-1 \mathrm{~V}$ potential range. These reversible features in the 
A) $\mathrm{pH} 1$

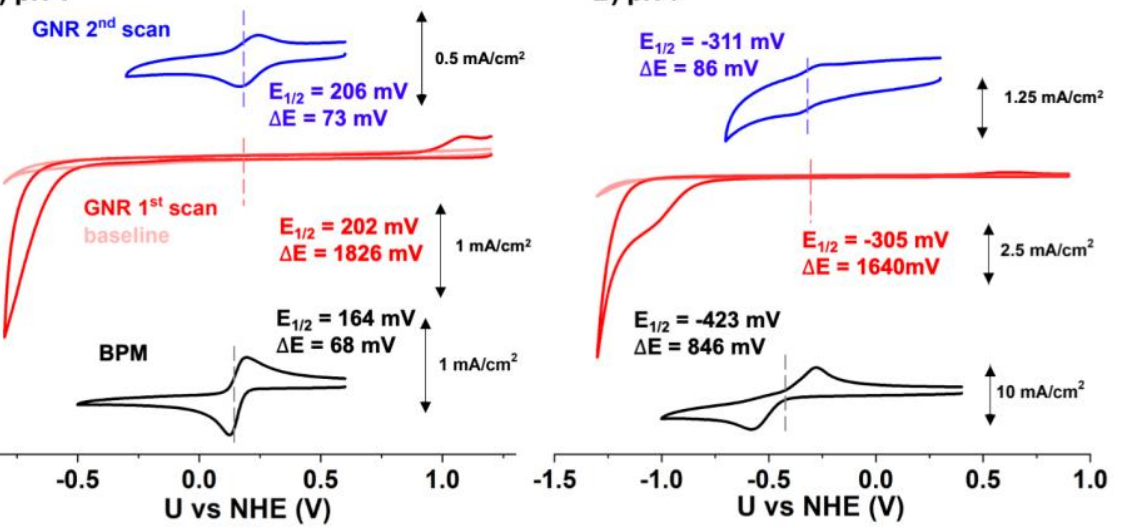

C) $\mathrm{pH} 13$

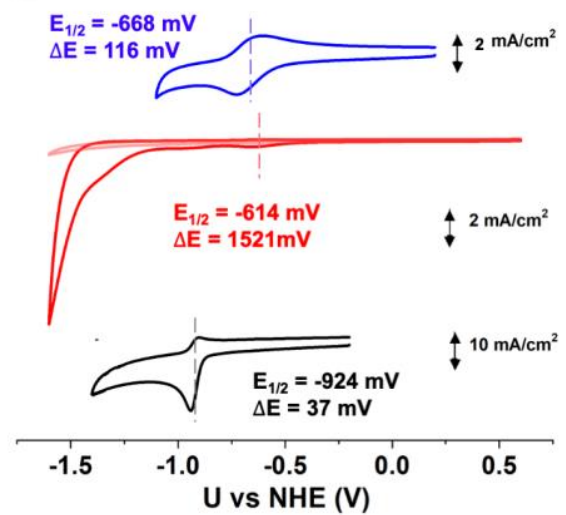

Figure 1. CVs of BPM (black), as-deposited GNR (red), and second-scan GNR (blue) in $1 \mathrm{M}$ aqueous solutions at pH 1 (left), pH 7 (middle), and pH 13 (right). GC working electrode; $100 \mathrm{mV} / \mathrm{s}$ scan rate. Background scans are shown in pale red in all three panels.

second scan appear only if the first scan ends at potentials more positive than the anodic process (Figure S10), suggesting that their formation requires reduction and re-oxidation of as-deposited GNR, and are assigned to the monolayer GNR deposited to the carbon support by electrochemical cycling. This assignment is based on the fact that the anodic-cathodic peak potential separation $\Delta \mathrm{E}$ is drastically reduced in the second-scan features, suggesting that the kinetics of electron and proton transfer are significantly improved. Furthermore, the integration of $\mathrm{CV}$ peaks is consistent with the expected current for the $0.5-3$ monolayers GNR coverage (Section S4). Based on these experimental findings, we hypothesize that the electrochemical reduction of as-deposited GNRs causes their partial desorption and solubilization in the solution above the electrode surface. The subsequent re-oxidation of solubilized GNRs results in their redeposition to the electrode surface, causing improved electronic coupling to the carbon electrode support (as illustrated in Figure 4). This simple electrochemical treatment provides a promising method towards deposition of molecular species to the carbon electrode and can be useful to the field of molecular enhancement of heterogeneous electrocatalysis. ${ }^{40}$

We now seek to answer the following question: does deposited GNR behave like the molecular species in solution or does it undergo the field-induced electrochemistry observed previously $^{23-25}$ for molecular species that are in strong electronic communication with the electrode? To address this, we compared Pourbaix diagrams for as-deposited GNRs (first CV scan) and monolayer GNRs (second CV scan). The Pourbaix diagram of as-deposited GNRs (Figure 2B) show that their half-wave potentials are slightly more positive than the corresponding potentials for BPM, and this shift is assigned to the extended conjugation of GNR. Aside from this difference, the Pourbaix diagrams of BPM and as-deposited GNR exhibit similar $\mathrm{pH}$-dependence, indicative of $\mathrm{GNRH}_{2}$ formation in the $\mathrm{pH}=4-14$ range and $\mathrm{GNRH}_{4}{ }^{2+}$ formation in the $0-4$ region (Scheme 1). The twoelectron, four-proton reduction behavior observed in the $\mathrm{pH}=0$ 4 region is indicative of weak electronic coupling between GNR and the carbon support. When strong electronic coupling is achieved, each proton transfer event generates a surface charge that is immediately compensated by the electron transfer from the electrode, leading to an overall neutral product and the 59 $\mathrm{mV} / \mathrm{pH}$ slope in the Pourbaix diagram. ${ }^{25}$ Again, the additional support of the PCET assignment in weakly-coupled GNR was provided by obtaining a good match between the experimental $E_{P C E T}^{0}$ values and those calculated using DFT (Tables S3 and S4 in Section S4B).

The Pourbaix diagram of the second CV-scan GNR (Figure 2C) is different from that obtained for as-deposited GNRs. In specific, the slope of the Pourbaix diagram remains the same throughout the entire $\mathrm{pH}=0-14$ range. The change in PCET chemistry that was observed at $\sim \mathrm{pH} 4$ in the case of BPM and as-deposited GNR was absent. We hypothesize that this behavior indicates that the second CV-scan GNR is strongly coupled with the carbon support. As mentioned, earlier, the stronglycoupled system is expected to only undergo processes that are overall charge neutral - each protonation of the surface nitrogen sites triggers the immediate charge compensation by electrons from the carbon electrode. ${ }^{23-25}$ Thus, the absence of the twoelectron, four-proton coupled chemistry in the acidic region is indicative of the strong coupling regime. The exact number of protons ( $\mathrm{m}$ ) and electrons (n) transferred during PCET is difficult to evaluate from Figure $2 \mathrm{C}$ because the $\sim 59 \mathrm{mV} / \mathrm{pH}$ slope informs only of the $\mathrm{m} / \mathrm{n}=1$ ratio. To obtain more insight into the details of the process, the Pourbaix diagram was treated using the model developed by Surendranath for field driven PCET. ${ }^{24,25}$ Based on this model, the $E_{P C E T}^{0}$ can be estimated from the potential of zero free charge for the electrode/electrolyte interface and the zero-field pKa values of the surface protonation sites. We applied this model to two possible PCET scenarios, one where two protons and electrons are transferred and another where four protons and electrons are transferred (Section S4B, SI). Better match with the experiment was observed for two proton/electron PCET, indicating the likelihood that only two nitrogen centers are protonated.

XPS investigations on modified carbon electrodes were conducted to provide additional insight into the electrochemical behavior of GNRs (experimental details are presented in Section S5). Electrodes were prepared by depositing GNR in different thickness regimes, to investigate both 

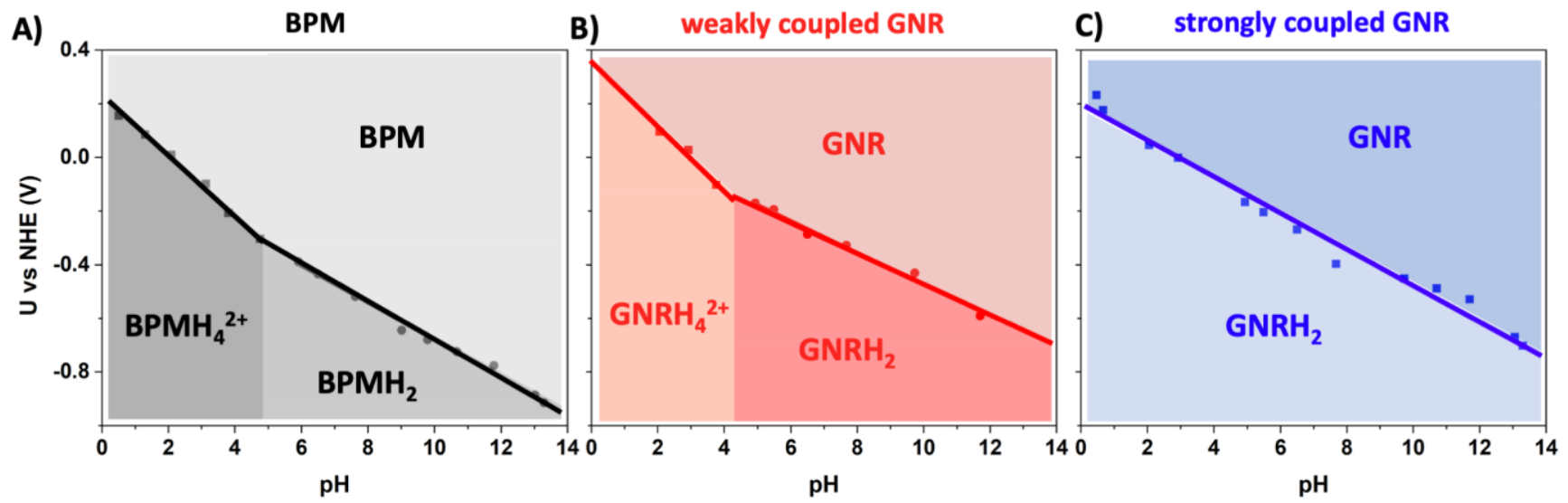

Figure 2. Pourbaix diagrams of A) BPM, B) GNR weakly coupled to the electrode, and C) GNR strongly coupled to the electrode. Shaded parts represent $\mathrm{pH}$-potential regions where annotated species are stable.

the weakly-coupled GNR aggregates and strongly-coupled GNR monolayers. The sample thickness had a profound effect on the observed N1s peaks (Figure 3A). Specifically, the thick sample showed a major peak at $398.3 \mathrm{eV}$ (red), which we attribute to pyridinic $\mathrm{N}$ atoms. ${ }^{35,41}$ The same peak was observed for GNR powder, indicating that it originates from GNR aggregates that are not coupled to the carbon electrode. The thick sample had additional higher binding energy (BE) features at 402.1 (blue) and $400.4 \mathrm{eV}$ (green). The $400.4 \mathrm{eV}$ feature was observed on the bare electrode, and it arises due to native nitrogen present in commercial carbon electrodes. ${ }^{42}$ The $402.1 \mathrm{eV}$ peak is assigned to the signal from monolayer GNR. This assignment is consistent with the appearance of the $\mathrm{N} 1$ s peak at higher $\mathrm{BE}$, a trend that is expected for GNR in intimate electronic interaction with the electron-withdrawing electrode environment. ${ }^{43,44}$ Meanwhile, the thin sample only shows features from the strongly-coupled monolayer GNR and the nitrogen present on the bare electrode (Figure 3A), further confirming our assignment of 398.3 and $402.1 \mathrm{eV}$ peaks to those arising from aggregate and monolayer GNRs, respectively.

The changes in the XPS N 1s peaks of thick GNR samples were monitored after electrochemical treatment (Figure 3B). The samples, initially at $\mathrm{OCP}$, were first reduced at a constant potential of $-1.7 \mathrm{~V}$, then re-oxidized at a constant potential of $+0.9 \mathrm{~V}$. The $\mathrm{N} 1 \mathrm{~s}$ XPS data were collected at three potentials to observe changes to the sample. Interestingly, cathodic treatment, which gives rise to PCET, does not lead to any significant shift in BE from the weakly- or strongly-coupled signals. The lack of the shift in the red signal after the $-1.7 \mathrm{~V}$ treatment is not surprising, as it is assigned to the residual GNR aggregates on the electrode which were not electrochemically reduced. We hypothesize that the reduced GNR detaches from the electrode and thus not contribute to the XPS signal. The lack of the shift in the blue signal is more surprising, as it originates from strongly-coupled GNRs which have participated in PCET. The observed insensitivity of the $\mathrm{N} 1 \mathrm{~s} \mathrm{BE}$ indicates that the effect of nitrogen protonation on $\mathrm{BE}$ is fully compensated by the electron density redistribution within the carbon electrode, resulting in a near zero BE shift (Figure 4). This finding is similar to in situ studies on strongly-coupled Rh-functionalized GC electrodes where, unlike their molecular analogs, no change in X-ray absorption near edge structure, i.e., oxidation state, during ioncoupled ET is observed. ${ }^{23,45}$ Similarly, subsequent anodic treatment does not induce changes to the binding energies of weakly- or strongly-coupled GNR. Importantly, integrated areas under XPS curves suggest the electrochemical treatment is enriching the ratio of strongly-to weakly-coupled GNRs as it changes from 1:12, 1:5, and 1:4 across the OCP, cathodic, and anodic panels, respectively. These results provide additional evidence that the electrochemical treatment of GNRs leads to the improved electronic contact between GNR and the carbon support.
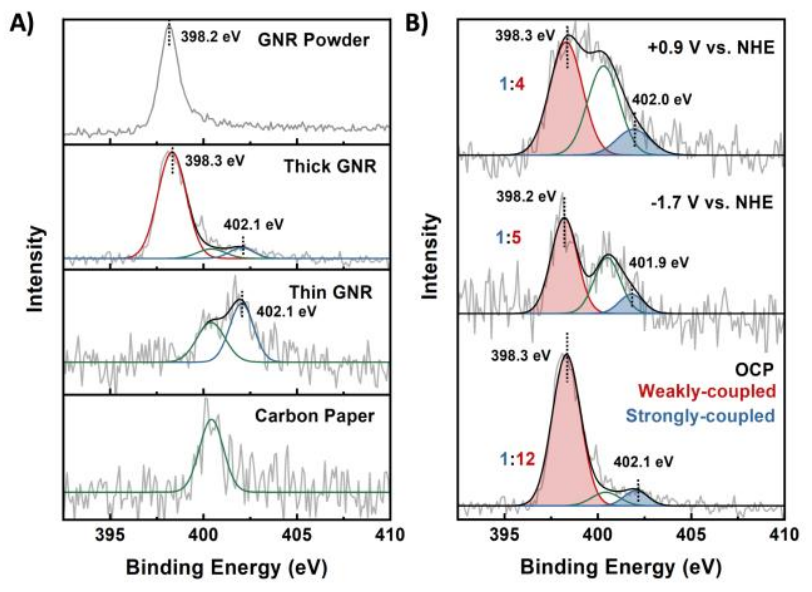

Figure 3. (A) XPS data recorded at open circuit potential (OCP) across multiple thickness regimes. Red, blue and green traces are used to identify the fitting of $\mathrm{N} 1 \mathrm{~s}$ from the weakly coupled GNR, strongly coupled GNR and carbon electrode, respectively. (B) Ex situ XPS data at OCP, cathodic and anodic potentials for thick GNR electrodes with ratios of strongly- and weakly-coupled GNR signals.

Based on combined electrochemical and XPS investigations, we hypothesize that strong coupling between GNR and the carbon support can be achieved via electrochemical cycling of drop-casted GNRs, as schematized for acidic electrolyte solution in Figure 4. Upon reduction (grey line, point 2, Figure 4), GNR is reduced to $\mathrm{GNRH}_{4}{ }^{2+}$, which detaches from the electrode and partially dissolves in the electrolyte solution. Subsequent oxidation to $\mathrm{GNRH}_{4}{ }^{2+}$ (point 3) redeposits some of the GNRs onto the electrode, now in improved electronic communication with GC, showing PCET behavior of a strongly-coupled system (points 4 and 5). 


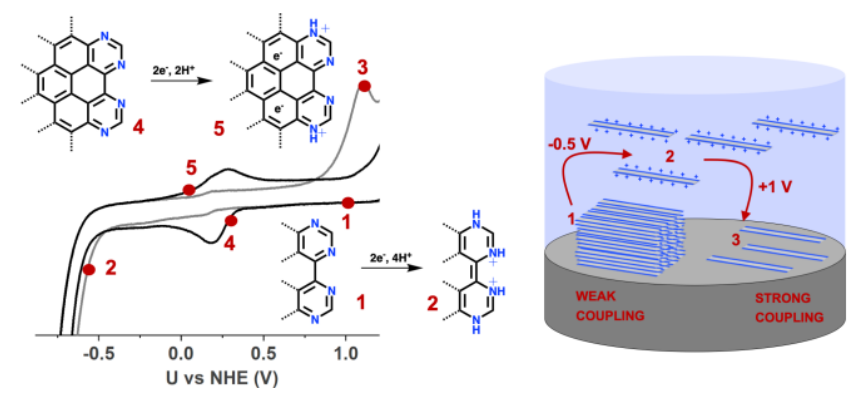

Figure 4. Electrochemical behavior of GNR on GC electrode. Gray like presents first $\mathrm{CV}$ scan, while black line is second scan at $\mathrm{pH} 1$. Red dot present certain points where transformation of interest occurs with insets showing corresponding relevant structural motifs.

In summary, we provide a mechanistic investigation of PCET in GNR/carbon electrode hybrids. Our work demonstrated that strong electronic coupling can be achieved between the molecular unit and the carbon support using non-covalent $\pi-\pi$ stacking interactions via simple electrochemical treatment that involves cathodic/anodic cycling of as-deposited GNR. The presence of nitrogen-containing functionalities on GNR opens up the possibility to coordinate transition metals and develop a new type of heterogeneous electrocatalysts with molecularlevel control of the catalytic units.

\section{ASSOCIATED CONTENT}

\section{Supporting Information}

Synthetic procedure, characterization of GNR, CVs, Pourbaix diagrams experimental and calculated data, XPS experimental and fitting details, reduction potential and $\mathrm{pKa}$ calculations, coordinates of optimized structures (PDF).

The Supporting Information is available free of charge on the ACS Publications website.

\section{AUTHOR INFORMATION}

\section{Corresponding Author}

*Ksenija D. Glusac: Department of Chemistry, University of Illinois at Chicago, Chicago, IL, glusac@uic.edu

\section{ACKNOWLEDGMENT}

This work was supported by the National Science Foundation grants 1954298 and 156597. We gratefully acknowledge the computing resources provided on Bebop, a high-performance computing cluster operated by the Laboratory Computing Resource Center at ANL.

\section{REFERENCES}

1. Cao, L.; Luo, Q.; Chen, J.; Wang, L.; Lin, Y.; Wang, H.; Liu, X.; Shen, X.; Zhang, W.; Liu, W., Dynamic oxygen adsorption on singleatomic Ruthenium catalyst with high performance for acidic oxygen evolution reaction. Nat. Commun. 2019, 10 (1), 1-9.

2. Xiao, M.; Zhu, J.; Li, G.; Li, N.; Li, S.; Cano, Z. P.; Ma, L.; Cui, P.; Xu, P.; Jiang, G., A single-atom iridium heterogeneous catalyst in oxygen reduction reaction. Angew. Chem. Int. Ed. 2019, 131 (28), 9742-9747.

3. Askins E. J.; Zoric M. R.; Li, K.; Luo, L.; Amine, K.; Glusac, K. D., Toward a mechanistic understanding of electrocatalytic nanocarbon. 2020.
4. McCrory, C. C.; Devadoss, A.; Ottenwaelder, X.; Lowe, R. D.; Stack, T. D. P.; Chidsey, C. E., Electrocatalytic $\mathrm{O}_{2}$ reduction by covalently immobilized mononuclear copper (I) complexes: evidence for a binuclear $\mathrm{Cu}_{2} \mathrm{O}_{2}$ intermediate. J Am. Chem. Soc. 2011, 133 (11), 36963699 .

5. Delamar, M.; Hitmi, R.; Pinson, J.; Saveant, J. M., Covalent modification of carbon surfaces by grafting of functionalized aryl radicals produced from electrochemical reduction of diazonium salts. J. Am. Chem. Soc. 1992, 114 (14), 5883-5884.

6. Willkomm, J.; Bertin, E.; Atwa, M.; Lin, J.-B.; Birss, V.; Piers, W. E., Grafting of a molecular rhenium $\mathrm{CO}_{2}$ reduction catalyst onto colloid-imprinted carbon. ACS Appl. Energy Mater. 2019, 2 (4), 24142418.

7. Li, H.; Cheng, F.; Duft, A. M.; Adronov, A., Functionalization of single-walled carbon nanotubes with well-defined polystyrene by “click" coupling. J. Am. Chem. Soc. 2005, 127 (41), 14518-14524.

8. Zhanaidarova, A.; Moore, C. E.; Gembicky, M.; Kubiak, C. P., Covalent attachment of [Ni (alkynyl-cyclam) ${ }^{2+}$ catalysts to glassy carbon electrodes. Chem. Commun. 2018, 54 (33), 4116-4119.

9. Zhanaidarova, A.; Ostericher, A. L.; Miller, C. J.; Jones, S. C.; Kubiak, C. P., Selective reduction of $\mathrm{CO}_{2}$ to $\mathrm{CO}$ by a molecular re (ethynyl-bpy) $(\mathrm{CO})_{3} \mathrm{Cl}$ catalyst and attachment to carbon electrode surfaces. Organometallics 2018, 38 (6), 1204-1207.

10. Jouikov, V.; Simonet, J., Novel method for grafting alkyl chains onto glassy carbon. Application to the easy immobilization of ferrocene used as redox probe. Langmuir 2012, 28 (1), 931-938.

11. Kallick, J. D.; Feng, W.-J.; McCrory, C. C., Controlled Formation of Multilayer Films of Discrete Molecular Catalysts for the Oxygen Reduction Reaction using a Layer-by-Layer Growth Mechanism Based on Sequential Click Chemistry. ACS Appl. Energy Mater. 2020. 3, 7, $6222-6231$.

12. Maurin, A.; Robert, M., Noncovalent immobilization of a molecular iron-based electrocatalyst on carbon electrodes for selective, efficient $\mathrm{CO}_{2}$-to-CO conversion in water. J. Am. Chem. Soc. 2016, 138 (8), 2492-2495.

13. Li, F.; Zhang, B.; Li, X.; Jiang, Y.; Chen, L.; Li, Y.; Sun, L., Highly efficient oxidation of water by a molecular catalyst immobilized on carbon nanotubes. Angew. Chem. Int. Ed. 2011, 50 (51), 12276-12279.

14. Blakemore, J. D.; Gupta, A.; Warren, J. J.; Brunschwig, B. S.; Gray, H. B., Noncovalent immobilization of electrocatalysts on carbon electrodes for fuel production. J. Am. Chem. Soc. 2013, 135 (49), 1828818291.

15. Das, A.; Stahl, S. S., Noncovalent immobilization of molecular electrocatalysts for chemical synthesis: efficient electrochemical alcohol oxidation with a pyrene-TEMPO conjugate. Angew. Chem. Int. Ed. 2017, 56 (30), 8892-8897.

16. Reuillard, B.; Ly, K. H.; Rosser, T. E.; Kuehnel, M. F.; Zebger, I.; Reisner, E., Tuning product selectivity for aqueous $\mathrm{CO} 2$ reduction with a Mn (bipyridine)-pyrene catalyst immobilized on a carbon nanotube electrode. J. Am. Chem. Soc. 2017, 139 (41), 14425-14435.

17. Jaegfeldt, H.; Kuwana, T.; Johansson, G., Electrochemical stability of catechols with a pyrene side chain strongly adsorbed on graphite electrodes for catalytic oxidation of dihydronicotinamide adenine dinucleotide. J. Am. Chem. Soc.1983, 105 (7), 1805-1814.

18. Creus, J.; Matheu, R.; Peñafiel, I.; Moonshiram, D.; Blondeau, P.; Benet-Buchholz, J.; García-Antón, J.; Sala, X.; Godard, C.; Llobet, A., A million turnover molecular anode for catalytic water oxidation. Angew. Chem. Int. Ed. 2016, 128 (49), 15608-15612.

19. Rajabi, S.; Ebrahimi, F.; Lole, G.; Odrobina, J.; Dechert, S.; Jooss, C.; Meyer, F., Water oxidizing diruthenium electrocatalysts immobilized on carbon nanotubes-effects of the number and positioning of pyrene anchors. ACS Catal. 2020. 10, 18, 10614-10626.

20. Toma, F. M.; Sartorel, A.; Iurlo, M.; Carraro, M.; Parisse, P.; Maccato, C.; Rapino, S.; Gonzalez, B. R.; Amenitsch, H.; Da Ros, T., Efficient water oxidation at carbon nanotube-polyoxometalate electrocatalytic interfaces. Nat. Chem. 2010, 2 (10), 826-831. 
21. Liu, Y.; McCrory, C. C., Modulating the mechanism of electrocatalytic $\mathrm{CO}_{2}$ reduction by cobalt phthalocyanine through polymer coordination and encapsulation. Nat. Commun. 2019, 10 (1), 1-10.

22. Thorogood, C. A.; Wildgoose, G. G.; Crossley, A.; Jacobs, R. M.; Jones, J. H.; Compton, R. G., Differentiating between ortho-and paraquinone surface groups on graphite, glassy carbon, and carbon nanotubes using organic and inorganic voltammetric and X-ray photoelectron spectroscopy labels. Chem. Mater. 2007, 19 (20), 4964-4974.

23. Jackson, M. N.; Kaminsky, C. J.; Oh, S.; Melville, J. F.; Surendranath, Y., Graphite conjugation eliminates redox intermediates in molecular electrocatalysis. J. Am. Chem. Soc. 2019, 141 (36), 1416014167.

24. Jackson, M. N.; Surendranath, Y., Molecular control of heterogeneous electrocatalysis through graphite conjugation. Acc. Chem. Res. 2019, 52 (12), 3432-3441.

25. Jackson, M. N.; Pegis, M. L.; Surendranath, Y., Graphite-conjugated acids reveal a molecular framework for proton-coupled electron transfer at electrode surfaces. ACS Cent. Sci. 2019, 5 (5), 831-841.

26. Wu, Y.; Jiang, Z.; Lu, X.; Liang, Y.; Wang, H., Domino electroreduction of $\mathrm{CO}_{2}$ to methanol on a molecular catalyst. Nature 2019, 575 (7784), 639-642.

27. Wang, X.; Sun, L.; Huang, Z.; Reddu, V.; Su, T.; Fisher, A. C., A planar, conjugated N4-macrocyclic cobalt complex for heterogeneous electrocatalytic $\mathrm{CO}_{2}$ reduction with high activity. Angew. Chem. Int. Ed. 2020. 59,17104-17109.

28. Zhang, X.; Wang, Y.; Gu, M.; Wang, M.; Zhang, Z.; Pan, W.; Jiang, Z.; Zheng, H.; Lucero, M.; Wang, H., Molecular engineering of dispersed nickel phthalocyanines on carbon nanotubes for selective $\mathrm{CO}_{2}$ reduction. Nat. Energy 2020, 5, 684-692.

29. Zhu, M.; Ye, R.; Jin, K.; Lazouski, N.; Manthiram, K., Elucidating the reactivity and mechanism of $\mathrm{CO}_{2}$ electroreduction at highly dispersed cobalt phthalocyanine. ACS Energy Lett. 2018, 3 (6), 13811386.

30. Zhang, X.; Wu, Z.; Zhang, X.; Li, L.; Li, Y.; Xu, H.; Li, X.; Yu, X.; Zhang, Z.; Liang, Y., Highly selective and active $\mathrm{CO}_{2}$ reduction electrocatalysts based on cobalt phthalocyanine/carbon nanotube hybrid structures. Nat. Commun. 2017, 8 (1), 1-8.

31. Hu, X. M.; Rønne, M. H.; Pedersen, S. U.; Skrydstrup, T.; Daasbjerg, K., Enhanced catalytic activity of cobalt porphyrin in $\mathrm{CO}_{2}$ electroreduction upon immobilization on carbon materials. Angew. Chem. Inter. Ed. 2017, 56 (23), 6468-6472.

32. Abdinejad, M.; Dao, C.; Deng, B.; Dinic, F.; Voznyy, O.; Zhang, X.-a.; Kraatz, H.-B., Electrocatalytic reduction of $\mathrm{CO}_{2}$ to $\mathrm{CH}_{4}$ and $\mathrm{CO}$ in aqueous solution using pyridine-porphyrins immobilized onto carbon nanotubes. ACS Sustain. Chem. Eng. 2020. 8, 25, 9549-9557.

33. Xu, F.; Zhang, L.; Ding, X.; Cong, M.; Jin, Y.; Chen, L.; Gao, Y., Selective electroreduction of dinitrogen to ammonia on a molecular iron phthalocyanine/O-MWCNT catalyst under ambient conditions. Chem. Commun. 2019, 55 (94), 14111-14114.
34. Sinha, S.; Zhang, R.; Warren, J. J., Low overpotential $\mathrm{CO}_{2}$ activation by a graphite-adsorbed cobalt porphyrin. ACS Catal. 2020, 10, 12284-12291.

35. Vo, T. H.; Perera, U. G. E.; Shekhirev, M.; Mehdi Pour, M.; Kunkel, D. A.; Lu, H.; Gruverman, A.; Sutter, E.; Cotlet, M.; Nykypanchuk, D., Nitrogen-doping induced self-assembly of graphene nanoribbon-based two-dimensional and three-dimensional metamaterials. Nano Lett. 2015, 15 (9), 5770-5777.

36. Morgenroth, F.; Reuther, E.; Müllen, K., Polyphenylene dendrimers: from three-dimensional to two-dimensional structures. Angew. Chem. Int. Ed. 1997, 36 (6), 631-634.

37. Vo, T. H.; Shekhirev, M.; Kunkel, D. A.; Morton, M. D.; Berglund, E.; Kong, L.; Wilson, P. M.; Dowben, P. A.; Enders, A.; Sinitskii, A., Large-scale solution synthesis of narrow graphene nanoribbons. Nat. Commun. 2014, 5 (1), 1-8.

38. Warren, J. J.; Tronic, T. A.; Mayer, J. M., Thermochemistry of proton-coupled electron transfer reagents and its implications. Chem. Rev. 2010, 110 (12), 6961-7001.

39. Choi, J.; Wagner, P.; Gambhir, S.; Jalili, R.; MacFarlane, D. R.; Wallace, G. G.; Officer, D. L., Steric modification of a cobalt phthalocyanine/graphene catalyst to give enhanced and stable electrochemical CO2 reduction to CO. ACS Energy Lett. 2019, 4 (3), 666-672.

40. Nam, D.-H.; De Luna, P.; Rosas-Hernández, A.; Thevenon, A.; Li, F.; Agapie, T.; Peters, J. C.; Shekhah, O.; Eddaoudi, M.; Sargent, E. H., Molecular enhancement of heterogeneous $\mathrm{CO}_{2}$ reduction. Nat. Mater. 2020, 19 (3), 266-276.

41. Sharifi, T.; Hu, G.; Jia, X.; Wagberg, T., Formation of active sites for oxygen reduction reactions by transformation of nitrogen functionalities in nitrogen-doped carbon nanotubes. ACS Nano 2012, 6 (10), 8904-8912.

42. Brunetti, B.; De Giglio, E.; Cafagna, D.; Desimoni, E., XPS analysis of glassy carbon electrodes chemically modified with 8-hydroxyquinoline-5-sulphonic acid. Surf. Interface Anal. 2012, 44 (4), 491-496.

43. Ramaswamy, N.; Tylus, U.; Jia, Q.; Mukerjee, S., Activity descriptor identification for oxygen reduction on nonprecious electrocatalysts: linking surface science to coordination chemistry. J. Am. Chem. Soc. 2013, 135 (41), 15443-15449.

44. Lazar, P.; Mach, R.; Otyepka, M., Spectroscopic fingerprints of graphitic, pyrrolic, pyridinic, and chemisorbed nitrogen in N-doped graphene. J. Phys. Chem. C 2019, 123 (16), 10695-10702.

45. Jackson, M. N.; Oh, S.; Kaminsky, C.; Chu, S. B.; Zhang, G.; Miller, J. T.; Surendranath, Y., Strong electronic coupling of molecular sites to graphitic electrodes via pyrazine conjugation. J. Am. Chem. Soc. 2018, 140, 1004-1010. 


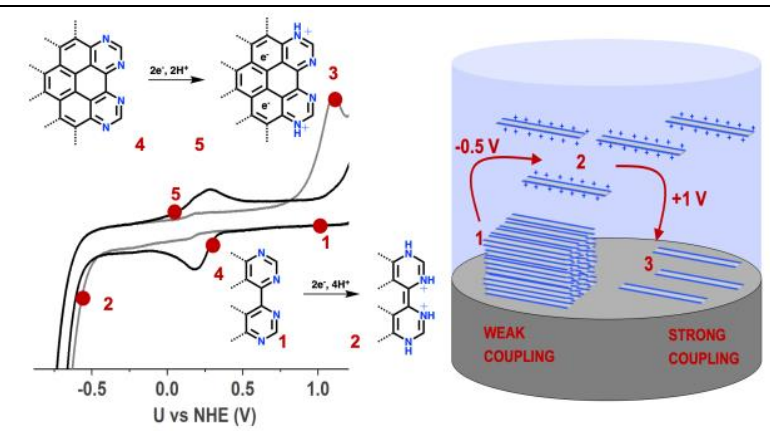

Table of Contents 